substitutions are found again to be less stable than the wild-type (Thr 157). The thermodynamic stabilities of the different lysozymes can be reasonably explained by the high-resolution crystal structures in terms of varying contributions of hydrogen bonding, side-chain flexibility, hydrophobic surface area and role of solvent water. However, even with this level of information we are still some way from being able to predict properties of novel proteins.

The book is on the whole well produced and edited. To reduce production costs, all the colour plates have been put in an appendix at the end, though black and white copies of the figures are also included in the main text. There is also a rather random and unexplained additional collection of colour plates which are not, as far as I could see, even mentioned in the main text of the book. The book is also not entirely free from typographical errors, but few readers will be misled by terms like 'synthotron radiation' or 'ransom coil [proteins]'. It was not the editors' aim to provide a text book, but rather to give an up-to-date review of the most important lines of research of use in protein design, and to this end the book is a success. One very useful feature is the list of key references at the end of each chapter which in most cases give the title of the paper (and for a novice in any of the diverse areas covered in the book, this is a great help).

Almost every contributor to the book mentions that though the field of protein engineering is in its infancy, the potential scientific, medicinal and commercial rewards are clear. Indeed it is notable that within the last five years almost every major pharmaceutical company has started a protein crystallographic group to explore structures of new genetically engineered proteins. This book is therefore very timely in providing a unique overview of this new multidisciplinary field and it can be readily recommended.

Malcolm D. Walkinshaw

\section{Pharmaceutical Division}

Sandoz $A G$

4002 Basel

Switzerland

Acta Cryst. (1988). B44, 320

\section{Book Received}

The following book has been received by the Editor. Brief and generally uncritical notices are given of works of marginal crystallographic interest; occasionally a book of fundamental interest is included under this heading because of difficulty in finding a suitable reviewer without delay.

High resolution NMR spectroscopy of synthetic polymers in bulk. Edited by Richard A. Komoroski. Pp. xi +379 . Deerfield Beach, Florida: VCH Publishers, 1987. Price $\$ 75.00$ (outside US and Canada from VCH VmbH, Weinheim, DM 185.00). This book is Volume 7 in the series Methods in Stereochemical Analysis. It contains ten essays. Following a general introduction, about half of the book deals with applications of ${ }^{13} \mathrm{C}$ NMR to amorphous materials below and above the glass-transition temperature and to semi-crystalline materials. The rest of the book is also concerned with applications of ${ }^{1} \mathrm{H}$ and ${ }^{2} \mathrm{H}$ spectra to such topics as conformational analysis and polymer motion. 